International Journal of Pure and Applied Mathematics

Volume 88 No. 1 2013, 15-34

ISSN: 1311-8080 (printed version); ISSN: 1314-3395 (on-line version)

url: http://www.ijpam.eu

doi: http://dx.doi.org/10.12732/ijpam.v88i1.2

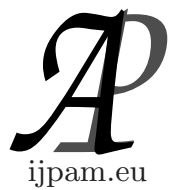

\title{
THE DETERMINANT OF AN INTERVAL MATRIX USING GAUSSIAN ELIMINATION METHOD
}

\author{
T. Nirmala ${ }^{1}$, D. Datta ${ }^{2}$, H.S. Kushwaha ${ }^{3}$, K. Ganesan ${ }^{4} \S$ \\ ${ }^{1,4}$ Department of Mathematics \\ Faculty of Engineering and Technology \\ SRM University \\ Kattankulathur, Chennai, 603203, INDIA \\ ${ }^{2,3}$ Health Physics Division \\ Bhabha Atomic Research Centre \\ Trombay, Mumbai, 400085, INDIA
}

\begin{abstract}
We introduce the notion of determinant and related results for interval matrices. We propose a Gaussian elimination like algorithm for computing the enclosures of the determinant of interval matrices. Numerical examples are also provided to show the efficiency of the proposed algorithm.
\end{abstract}

AMS Subject Classification: 15A09, 65F05, 65G30

Key Words: interval number, interval arithmetic, interval matrix, determinant, Gaussian algorithm

\section{Introduction}

To model real-life problems and perform computations we must deal with uncertainty and inexactness. These uncertainty and inexactness are due to measurement inaccuracy, simplification of physical models, variations of the parameters of the system, computational errors etc. Interval analysis is an efficient and reliable tool that allows us to handle such problems effectively. Determinants of interval matrices plays a key role in the study of Eigen values and eigenvectors.

Received: May 28, 2013

(c) 2013 Academic Publications, Ltd.

$\S$ Correspondence author url: www.acadpubl.eu 
Eigen values of general interval matrices are useful to study robust stability margins of analog and discrete systems and convergence rates in numerical analysis. Determinants of real symmetric and Hermitian interval matrices play a major role in signal processing, control, stochastic processes and statistics. Evaluation of determinants of matrices in a straightforward way from the definition, requires about $n . n$ ! arithmetic operations for an $(n \times n)$ matrix, where as Gaussian elimination method requires only $\frac{2 n^{3}}{3}$ operations which is very much less when compared to the number n.n!. Hence we propose a Gaussian elimination like algorithm for computing determinants of interval matrices.

Let $\mathbf{I R}=\left\{\tilde{a}=\left[a_{1}, a_{2}\right]: a_{1} \leq a_{2}\right.$ and $\left.a_{1}, a_{2} \in \mathbf{R}\right\}$ be the set of all proper intervals and $\overline{\mathbf{I R}}=\left\{\tilde{a}=\left[a_{1}, a_{2}\right]: a_{1}>a_{2}\right.$ and $\left.a_{1}, a_{2} \in \mathbf{R}\right\}$ be the set of all improper intervals on the real line $\mathbf{R}$. If $a_{1}=a_{2}=a$, then $\tilde{a}=[a, a]$ is a real number (or a degenerate interval). We shall use the terms "interval" and "interval number" interchangeably. The mid-point and width (or half-width) of an interval number $\tilde{a}=\left[a_{1}, a_{2}\right]$ are defined as $m(\tilde{a})=\left(\frac{a_{1}+a_{2}}{2}\right)$ and $w(\tilde{a})=\left(\frac{a_{2}-a_{1}}{2}\right)$. We denote the set of generalized intervals (proper and improper) by $\mathbf{D}=\mathbf{I R} \cup \overline{\mathbf{I R}}=\left\{\left[a_{1}, a_{2}\right]: a_{1}, a_{2} \in \mathbf{R}\right\}$. The set of generalized intervals $\mathbf{D}$ is a group with respect to addition and multiplication operations, while maintaining the inclusion monotonicity.

The "dual" is an important monadic operator proposed by Kaucher [9] that reverses the end-points of the intervals and expresses an element-to-element symmetry between proper and improper intervals in $\mathbf{D}$. For $\tilde{a}=\left[a_{1}, a_{2}\right] \in \mathbf{D}$, its dual is defined by $\operatorname{dual}(\tilde{a})=\operatorname{dual}\left[a_{1}, a_{2}\right]=\left[a_{2}, a_{1}\right]$. The opposite of an interval $\tilde{a}=\left[a_{1}, a_{2}\right]$ is $\operatorname{opp}\left\{\left[a_{1}, a_{2}\right]\right\}=\left[-a_{1},-a_{2}\right]$ which is the additive inverse of $\left[a_{1}, a_{2}\right]$ and $\left[\frac{1}{a_{1}}, \frac{1}{a_{2}}\right]$ is the multiplicative inverse of $\left[a_{1}, a_{2}\right]$, provided $m\left(\left[a_{1}, a_{2}\right]\right)=\left(\frac{a_{1}+a_{2}}{2}\right) \neq 0$.

That is $\quad \tilde{a}+(-\operatorname{dual} \tilde{a})=\tilde{a}-\operatorname{dual}(\tilde{a})=\left[a_{1}, a_{2}\right]-\operatorname{dual}\left(\left[a_{1}, a_{2}\right]\right)$ $=\left[a_{1}, a_{2}\right]-\left[a_{2}, a_{1}\right]=\left[a_{1}-a_{1}, a_{2}-a_{2}\right]=[0,0]$

and $\quad \tilde{a} \times\left(\frac{1}{\text { dual } \tilde{a}}\right)=\left[a_{1}, a_{2}\right] \times\left(\frac{1}{\operatorname{dual}\left(\left[a_{1}, a_{2}\right]\right)}\right)=\left[a_{1}, a_{2}\right] \times \frac{1}{\left[a_{2}, a_{1}\right]}$ $=\left[a_{1}, a_{2}\right] \times\left[\frac{1}{a_{1}}, \frac{1}{a_{2}}\right]=\left[\frac{a_{1}}{a_{1}}, \frac{a_{2}}{a_{2}}\right]=[1,1]$

In this paper we use the generalized interval arithmetic proposed by Nirmala 
et.al [16] by extending Kauchers [9] interval arithmetic.This arithmetic operation satisfying group properties with respect to addition and multiplication operations and satisfying the distributive relations between intervals, while maintaining the inclusion monotonicity.

The rest of this paper is organized as follows: In Section 2, we extend the Sengupta and Pal's [2] method of comparison of interval numbers to the set of all generalized intervals $\mathbf{D}$. We recall the generalized interval arithmetic $\mathbf{D}$ on the set of generalized interval numbers $\mathbf{D}$ proposed by Nirmala et.al [16]. In Section 3, we introduce the notion of interval matrices and the arithmetic operations on interval matrices. We define the determinant of square interval matrices and discuss some of its properties. In Section 4, we propose a Gaussian elimination like algorithm for computing the enclosures of the determinant of interval matrices. Numerical examples are also provided to show the efficiency of the proposed algorithm.

\section{Preliminaries}

The aim of this section is to present some notations, notions and results which are of useful in our further considerations.

\subsection{Comparing Interval Numbers}

Sengupta and Pal [2] proposed a simple and efficient index for comparing any two intervals on IR through decision maker's satisfaction. We extend this concept to the set of all generalized intervals on $\mathbf{D}$.

Definition 1. Let $\preceq$ be an extended order relation between the interval numbers $\tilde{a}=\left[a_{1}, a_{2}\right]$ and $\tilde{b}=\left[b_{1}, b_{2}\right]$ in $\mathbf{D}$, then for $m(\tilde{a})<m(\tilde{b})$, we construct a premise $(\tilde{a} \preceq \tilde{b}$ ) which implies that $\tilde{a}$ is inferior to $\tilde{b}$ (or $\tilde{b}$ is superior to $\tilde{a}$ ). Here, the term 'inferior to' ('superior to') is analogous to 'less than' ('greater than').

Definition 2. An acceptability function $\mathcal{A}_{\preceq}: \mathbf{D} \times \mathbf{D} \longrightarrow[0, \infty)$ is defined as: $\mathcal{A}_{\preceq}(\tilde{a}, \tilde{b})=\mathcal{A}(\tilde{a} \preceq \tilde{b})=\frac{(m(\tilde{b})-m(\tilde{a}))}{(w(\tilde{b})+w(\tilde{a}))}$, where $w(\tilde{b})+w(\tilde{a}) \neq 0$. $\mathcal{A}_{\preceq}$ may be interpreted as the grade of acceptability of the 'first interval number $\tilde{a}$ to be inferior to the second interval number $\tilde{b}$.

For any two interval numbers $\tilde{a}$ and $\tilde{b}$ in $\mathbf{D}$, either $\mathcal{A}(\tilde{a} \preceq \tilde{b})>0$ or $\mathcal{A}(\tilde{b} \preceq$ $\tilde{a})>0$ or $\mathcal{A}(\tilde{a} \preceq \tilde{b})=\mathcal{A}(\tilde{b} \preceq \tilde{a})=0$ and $\mathcal{A}(\tilde{a} \preceq \tilde{b})+\mathcal{A}(\tilde{b} \preceq \tilde{a})=0$. Also the 
proposed $\mathcal{A}$-index is transitive; for any three interval numbers $\tilde{a}, \tilde{b}$ and $\tilde{c}$ in $\mathbf{D}$, if $\mathcal{A}(\tilde{a} \preceq \tilde{b}) \geq 0$ and $\mathcal{A}(\tilde{b} \preceq \tilde{c}) \geq 0$, then $\mathcal{A}(\tilde{a} \preceq \tilde{c}) \geq 0$. But it does not mean that $\mathcal{A}(\tilde{a} \preceq \tilde{c}) \geq \max \{\mathcal{A}(\tilde{a} \preceq \tilde{b}), \mathcal{A}(\tilde{b} \preceq \tilde{c})\}$. If $\mathcal{A}(\tilde{a} \preceq \tilde{b})=0$, then we say that the interval numbers $\tilde{a}$ and $\tilde{b}$ are equivalent (or non-inferior to each other) and we denote it by $\tilde{a} \approx \tilde{b}$. In particular, whenever $\mathcal{A}(\tilde{a} \preceq \tilde{b})=0$ and $w(\tilde{a})=w(\tilde{b})$, then $\tilde{a}=\tilde{b}$. Also if $\mathcal{A}(\tilde{a} \preceq \tilde{b}) \geq 0$, then we say that $\tilde{a} \preceq \tilde{b}$ and if $\mathcal{A}(\tilde{b} \preceq \tilde{a}) \geq 0$, then we say that $\tilde{b} \preceq \tilde{a}$.

Remark 3. For any two interval numbers $\tilde{a}, \tilde{b} \in \mathbf{D}$, we have $\mathcal{A}(\tilde{a} \preceq$ $\tilde{b})+\mathcal{A}(\tilde{b} \preceq \tilde{a})=0$. If $m(\tilde{a})=0$ then we say that $\tilde{a}$ is a zero interval number. In particular, if $m(\tilde{a})=0$ and $w(\tilde{a})=0$, then $\tilde{a}=[0,0]$. Also, if $m(\tilde{a})=0$ and $w(\tilde{a}) \neq 0$, then $\tilde{a} \approx \tilde{0}$. It is to be noted that if $\tilde{a}=[0,0]=\tilde{0}$, then $\tilde{a} \approx \tilde{0}$, but the converse need not be true. If $\tilde{a} \not \approx \tilde{0}$ (i.e. $\tilde{a}$ is not equivalent to $\tilde{0}$ ), then $\tilde{a}$ is said to be a non-zero interval number. It is to be noted that if $\tilde{a} \not \tilde{0}$, then $\tilde{a} \neq \tilde{0}$, but the converse need not be true. If $\mathcal{A}(\tilde{a} \succeq \tilde{0}) \geq 0$ and $\mathcal{A}(\tilde{a} \succeq \tilde{0}) \neq 0$, that is, if $\tilde{a} \succeq \tilde{0}$ and $\tilde{a} \not \approx \tilde{0}$, then $\tilde{a}$ is said to be a positive interval number and is denoted by $\tilde{a} \succ \tilde{0}$.

\subsection{A New Interval Arithmetic}

Ganesan and Veeramani [4] proposed a new interval arithmetic on IR. We extend this arithmetic operations to the set of generalized interval numbers $\mathbf{D}$ and incorporating the concept of dual. For $\tilde{a}=\left[a_{1}, a_{2}\right], \tilde{b}=\left[b_{1}, b_{2}\right] \in \mathbf{D}$ and for $* \in\{+,-, \cdot, \div\}$, we define $\tilde{a} * \tilde{b}=[m(\tilde{a}) * m(\tilde{b})-k, m(\tilde{a}) * m(\tilde{b})+k]$, where $k=\min \{(m(\tilde{a}) * m(\tilde{b}))-\alpha, \beta-(m(\tilde{a}) * m(\tilde{b}))\}, \alpha$ and $\beta$ are the end points of the interval $\tilde{a} \odot \tilde{b}$ under the existing interval arithmetic. In particular

(i) Addition:

$$
\begin{aligned}
& \tilde{a}+\tilde{b}=\left[a_{1}, a_{2}\right]+\left[b_{1}, b_{2}\right]=[(m(\tilde{a})+m(\tilde{b}))-k,(m(\tilde{a})+m(\tilde{b}))+k], \\
& \text { where } k=\left\{\frac{\left(b_{2}+a_{2}\right)-\left(b_{1}+a_{1}\right)}{2}\right\} .
\end{aligned}
$$

(ii) Subtraction:

$$
\begin{aligned}
\tilde{a}-\tilde{b} & =\left[a_{1}, a_{2}\right]-\left[b_{1}, b_{2}\right]=[(m(\tilde{a})-m(\tilde{b}))-k,(m(\tilde{a})-m(\tilde{b}))+k] \\
\text { where } k & =\left\{\frac{\left(b_{2}+a_{2}\right)-\left(b_{1}+a_{1}\right)}{2}\right\} .
\end{aligned}
$$


Also if $\tilde{a}=\tilde{b}$, i.e. if $\left[a_{1}, a_{2}\right]=\left[b_{1}, b_{2}\right]$, then

$$
\begin{aligned}
\tilde{a}-\tilde{b} & =\tilde{a}-\operatorname{dual}(\tilde{a})=\left[a_{1}, a_{2}\right]-\left[a_{2}, a_{1}\right] \\
& =\left[a_{1}-a_{1}, a_{2}-a_{2}\right]=[0,0]
\end{aligned}
$$

(iii) Multiplication:

$$
\tilde{a} . \tilde{b}=\tilde{a} \tilde{b}=\left[a_{1}, a_{2}\right]\left[b_{1}, b_{2}\right]=[m(\tilde{a}) m(\tilde{b})-k, m(\tilde{a}) m(\tilde{b})+k],
$$

where $k=\min \{(m(\tilde{a}) m(\tilde{b}))-\alpha, \beta-(m(\tilde{a}) m(\tilde{b}))\}$,

$\alpha=\min \left(a_{1} b_{1}, a_{1} b_{2}, a_{2} b_{1}, a_{2} b_{2}\right)$ and $\beta=\max \left(a_{1} b_{1}, a_{1} b_{2}, a_{2} b_{1}, a_{2} b_{2}\right)$.

(iv) Division: $1 \div \tilde{a}=\frac{1}{\tilde{a}}=\frac{1}{\left[a_{1}, a_{2}\right]}=\left[\frac{1}{m(\tilde{a})}-k, \frac{1}{m(\tilde{a})}+k\right]$, where

$$
k=\min \left\{\frac{1}{a_{2}}\left(\frac{a_{2}-a_{1}}{a_{1}+a_{2}}\right), \frac{1}{a_{1}}\left(\frac{a_{2}-a_{1}}{a_{1}+a_{2}}\right)\right\} \text { and } 0 \notin\left[a_{1}, a_{2}\right] .
$$

Also if $\tilde{a}=\tilde{b}$ i.e. $\left[a_{1}, a_{2}\right]=\left[b_{1}, b_{2}\right]$, then

$$
\begin{aligned}
\frac{\tilde{a}}{\tilde{b}} & =\frac{\tilde{a}}{\tilde{a}}=\frac{\tilde{a}}{\operatorname{dual}(\tilde{a})}=\left[a_{1}, a_{2}\right] \cdot \frac{1}{\left[a_{2}, a_{1}\right]} \\
& =\left[a_{1}, a_{2}\right] \cdot\left[\frac{1}{a_{1}}, \frac{1}{a_{2}}\right]=\left[\frac{a_{1}}{a_{1}}, \frac{a_{2}}{a_{2}}\right]=[1,1]
\end{aligned}
$$

From (iii), it is clear that $\lambda \tilde{a}= \begin{cases}{\left[\lambda a_{1}, \lambda a_{2}\right],} & \text { for } \lambda \geq 0 \\ {\left[\lambda a_{2}, \lambda a_{1}\right],} & \text { for } \lambda<0 .\end{cases}$

It is to be noted that we use $\odot$ to denote the existing interval arithmetic and $*$ to denote the modified interval arithmetic. But wherever there is no confusion we use the same notation for both the cases. It is also to be noted that $\tilde{a} * \tilde{b} \subseteq \tilde{a} \odot \tilde{b}=\{a \odot b / a \in \tilde{a}, b \in \tilde{b}\}$, where $\odot \in\{\oplus, \ominus, \otimes, \oslash\}$ is the existing interval arithmetic. For example if $\tilde{a}=[-1,2]$ and $\tilde{b}=[3,5]$, then $\tilde{a} \otimes \tilde{b}=[-1,2] \otimes[3,5]=[\min (-3,-5,6,10), \max (-3,-5,6,10)]=[-5,10]$ and $\tilde{a} * \tilde{b}=\tilde{a} \tilde{b}=[-1,2][3,5]=[-5,9]$ so that $\tilde{a} * \tilde{b} \subseteq \tilde{a} \odot \tilde{b}$. It is also important to note that by using this modified interval arithmetic we are able to prove the distributive law for interval numbers and hence many other important results. 


\section{Main Results}

An interval matrix $\tilde{A}$ is a matrix whose elements are interval numbers. An interval matrix $\tilde{A}$ will be written as: $\tilde{A}=\left(\begin{array}{ccc}\tilde{a}_{11} & \cdots & \tilde{a}_{1 n} \\ \cdots & \cdots & \cdots \\ \tilde{a}_{m 1} & \cdots & \tilde{a}_{m n}\end{array}\right)=\left(\tilde{a}_{i j}\right)_{1 \leq i \leq m, 1 \leq j \leq n}$, where each $\tilde{a}_{i j}=\left[\underline{a}_{i j}, \bar{a}_{i j}\right]$ (or) $\tilde{A}=[\underline{A}, \bar{A}]$ for some $\underline{A}, \bar{A}$ satisfying $\underline{A} \leq \bar{A}$. We use $\mathbf{D}^{m \times n}$ to denote the set of all $(m \times n)$ interval matrices. The midpoint (center) of an interval matrix $\tilde{A}$ is the matrix of midpoints of its interval elements defined as $m(\tilde{A})=\left(\begin{array}{ccc}m\left(\tilde{a}_{11}\right) & \ldots & m\left(\tilde{a}_{1 n}\right) \\ \ldots & \ldots & \ldots \\ m\left(\tilde{a}_{m 1}\right) & \ldots & m\left(\tilde{a}_{m n}\right)\end{array}\right) \cdot$ The width of an interval matrix $\tilde{A}$ is the matrix of widths of its interval elements defined as $w(\tilde{A})=\left(\begin{array}{ccc}w\left(\tilde{a}_{11}\right) & \cdots & w\left(\tilde{a}_{1 n}\right) \\ \cdots & \cdots & \cdots \\ w\left(\tilde{a}_{m 1}\right) & \cdots & w\left(\tilde{a}_{m n}\right)\end{array}\right)$ which is always nonnegative. We use $O$ to denote the null matrix $\left(\begin{array}{ccc}0 & \ldots & 0 \\ \cdots & \ldots & \ldots \\ 0 & \ldots & 0\end{array}\right)$ and $\tilde{O}$ to denote the null interval matrix $\left(\begin{array}{ccc}\tilde{0} & \ldots & \tilde{0} \\ \ldots & \ldots & \ldots \\ \tilde{0} & \ldots & \tilde{0}\end{array}\right)$. Also we use $I$ to denote the identity matrix $\left(\begin{array}{ccc}1 & \cdots & 0 \\ \cdots & 1 & \cdots \\ 0 & \cdots & 1\end{array}\right)$ and $\tilde{I}$ to denote the identity interval matrix $\left(\begin{array}{ccc}\tilde{1} & \ldots & \tilde{0} \\ \ldots & \tilde{1} & \ldots \\ \tilde{0} & \ldots & \tilde{1}\end{array}\right)$. If $m(\tilde{A})=m(\tilde{B})$, then the interval matrices $\tilde{A}$ and $\tilde{B}$ are said to be equivalent and is denoted by $\tilde{A} \approx \tilde{B}$. In particular if $m(\tilde{A})=$ $m(\tilde{B})$ and $w(\tilde{A})=w(\tilde{B})$, then $\tilde{A}=\tilde{B}$. If $m(\tilde{A})=O$, then we say that $\tilde{A}$ is a zero interval matrix. In particular if $m(\tilde{A})=O$ and $w(\tilde{A})=O$, then $\tilde{A}=\left(\begin{array}{ccc}{[0,0]} & \ldots & {[0,0]} \\ \ldots & \ldots & \ldots \\ {[0,0]} & \ldots & {[0,0]}\end{array}\right) \cdot$ Also, if $m(\tilde{A})=O$ and $w(\tilde{A}) \neq O$, then $\tilde{A}=\left(\begin{array}{ccc}\tilde{0} & \cdots & \tilde{0} \\ \cdots & \cdots & \cdots \\ \tilde{0} & \cdots & \tilde{0}\end{array}\right) \approx \tilde{O}$. If $\tilde{A} \not \tilde{O}$ (i.e. $\tilde{A}$ is not equivalent to $\tilde{O}$ ), then $\tilde{A}$ is said to be a non-zero interval matrix. If $m(\tilde{A})=I$, then we say that $\tilde{A}$ is a identity interval matrix. In particular if $m(\tilde{A})=I$ and $w(\tilde{A})=O$, 
then $\tilde{A}=\left(\begin{array}{ccc}{[1,1]} & \cdots & {[0,0]} \\ \cdots & {[1,1]} & \ldots \\ {[0,0]} & \ldots & {[1,1]}\end{array}\right)$. Also, if $m(\tilde{A})=I$ and $w(\tilde{A}) \neq O$, then
$\tilde{A}=\left(\begin{array}{ccc}\tilde{1} & \cdots & \tilde{0} \\ \ldots & \tilde{1} & \ldots \\ \tilde{0} & \ldots & \tilde{1}\end{array}\right) \approx \tilde{I}$.

\subsection{Arithmetic Operations on Interval Matrices}

We introduce the following arithmetic operations on interval matrices. As with interval numbers, we define the arithmetic operations on interval matrices as follows. If $\tilde{A}, \tilde{B} \in \mathbf{D}^{m \times n}, \tilde{\mathbf{x}} \in \mathbf{D}^{n}$ and $\tilde{\alpha} \in \mathbf{D}$, then

(i). $\tilde{\alpha} \tilde{A} \approx\left(\tilde{\alpha} \tilde{a}_{i j}\right)_{1 \leq i \leq m, 1 \leq j \leq n}$

(ii). $\tilde{A}+\tilde{B} \approx\left(\tilde{a}_{i j}+\tilde{b}_{i j}\right)_{1 \leq i \leq m, 1 \leq j \leq n}$

(iii). $\quad \tilde{A}-\tilde{B} \approx \begin{cases}\left(\tilde{a}_{i j}-\tilde{b}_{i j}\right)_{1 \leq i \leq m, 1 \leq j \leq n}, & \text { if } \tilde{A} \not \approx \tilde{B} \\ \tilde{A}-\operatorname{dual}(\tilde{A}) \approx \tilde{O}=O, & \text { if } \tilde{A} \approx \tilde{B}\end{cases}$

(iv). $\tilde{A} \tilde{B} \approx\left(\sum_{k=1}^{n} \tilde{a}_{i k} \tilde{b}_{k j}\right)_{1 \leq i \leq m, 1 \leq j \leq n}$

(v). $\tilde{A} \tilde{\mathbf{x}} \approx\left(\sum_{j=1}^{n} \tilde{a}_{i j} \tilde{\mathbf{x}}\right)_{1 \leq i \leq m}$

\subsection{Determinant of Interval Matrices}

We define the determinant of a square interval matrix as in the case of real square matrix except that the determinant of an interval matrix is an interval number. That is $\operatorname{det} \tilde{A}=|\tilde{A}|=\sum \tilde{a}_{i j} \tilde{A}_{i j}$, where $\tilde{A}_{i j}$ with usual meaning. It is easy to see that most of the properties of determinants of classical matrices are hold good (up to equivalent) for the determinants of interval matrices under the modified interval arithmetic.

\subsection{Properties of Determinants}

Let $\tilde{A}$ be an interval matrix of order $(n \times n)$. Then

1. $\operatorname{det} \tilde{A} \approx \operatorname{det} \tilde{A^{T}}$, for all $(n \times n)$ interval matrices $\tilde{A}$. 
2. If one row of $\tilde{A}$ consists entirely of zero fuzzy numbers, then $\tilde{A} \approx \tilde{0}$.

3. If $\tilde{B}$ is an interval matrix obtained from $\tilde{A}$ by multiplying one row of $\tilde{A}$ by the scalar $\lambda$ then $\operatorname{det} \tilde{B} \approx \lambda \operatorname{det} \tilde{A}$.

4. If $\tilde{B}$ is an interval matrix obtained from $\tilde{A}$ by interchanging two rows of $\tilde{A}$ then $\operatorname{det} \tilde{B} \approx-\operatorname{det} \tilde{A}$.

5. If two rows of $\tilde{A}$ are identical, then $\operatorname{det} \tilde{A} \approx \tilde{0}$.

Theorem 4. If $\tilde{A}$ is an $(n \times n)$ upper triangular or lower triangular interval matrix, then $|\tilde{A}|$ is equivalent to the product of the elements in its main diagonal.

Proof. Let $\tilde{A}$ be an upper triangular interval matrix of order $(n \times n)$. The proof is by induction on $n$.Let $\tilde{A}=\left(\begin{array}{cc}\tilde{a}_{11} & \tilde{a}_{12} \\ \tilde{0} & \tilde{a}_{22}\end{array}\right)=\left(\begin{array}{cc}{\left[\underline{a}_{11}, \bar{a}_{11}\right]} & {\left[\underline{a}_{12}, \bar{a}_{12}\right]} \\ {[0,0]} & {\left[\underline{a}_{22}, \bar{a}_{22}\right]}\end{array}\right)$. Then by definition

$$
\begin{aligned}
|\tilde{A}| & \approx \tilde{a}_{11} \tilde{a}_{22} \approx\left[\underline{a}_{11}, \bar{a}_{11}\right]\left[\underline{a}_{22}, \bar{a}_{22}\right] \\
& \approx \text { the product of the entries in the leading diagonal of } \tilde{A} .
\end{aligned}
$$

Hence the determinant of a $(2 \times 2)$ upper triangular interval matrix, is the product of the entries in its leading diagonal. Therefore the result is true for $n=2$. Assume that the result is true for $n=k$, ie.

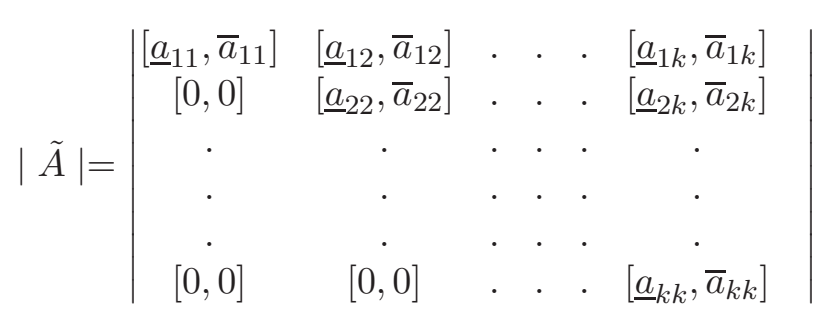

Now for $n=k+1$, determinant of upper triangular interval matrix $\tilde{A}$ of size $(k+1)$ by $(k+1)$ by expanding along the bottom row (since all entries in the bottom row are zero except $\left.\left[\underline{a}_{k+1}, \bar{a}_{k+1}\right]\right)$, we have

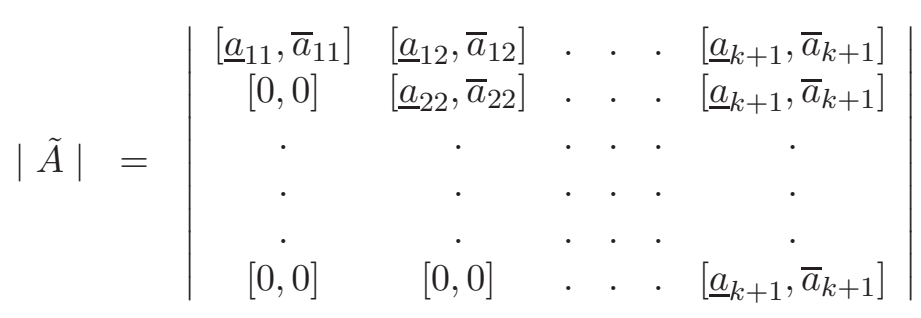




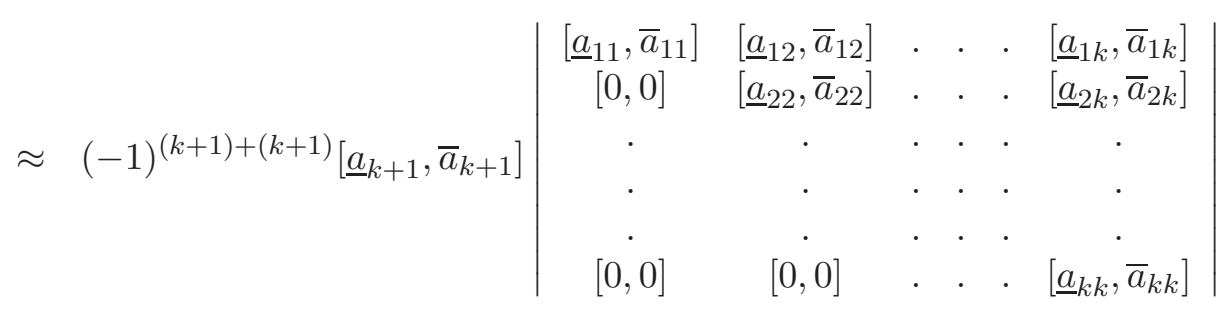

$$
\begin{aligned}
|\tilde{A}| & \approx(-1)^{(2 k+2)}\left[\underline{a}_{k+1}, \bar{a}_{k+1}\right]\left[\underline{a}_{11}, \bar{a}_{11}\right]\left[\underline{a}_{22}, \bar{a}_{22}\right] \ldots\left[\underline{a}_{k k}, \bar{a}_{k k}\right], \text { by equation }(2) \\
& \approx(-1)^{2(k+1)}\left[\underline{a}_{11}, \bar{a}_{11}\right]\left[\underline{a}_{22}, \bar{a}_{22}\right] \ldots\left[\underline{a}_{k k}, \bar{a}_{k k}\right]\left[\underline{a}_{k+1}, \bar{a}_{k+1}\right] \\
& \approx\left[\underline{a}_{11}, \bar{a}_{11}\right]\left[\underline{a}_{22}, \bar{a}_{22}\right] \ldots\left[\underline{a}_{k k}, \bar{a}_{k k}\right]\left[\underline{a}_{k+1}, \bar{a}_{k+1}\right], \text { since }(-1)^{2(k+1)}=1 . \\
& \approx \text { the product of the entries in the leading diagonal of } \tilde{A} .
\end{aligned}
$$

Therefore the result is true for $n=k+1$ also. Hence the theorem. If $\tilde{A}$ is lower triangular interval matrix, then the theorem can also be proved in the similar way.

Theorem 5. If $\tilde{B}$ is an interval matrix obtained from $\tilde{A}$ by adding a multiple of one row of $\tilde{A}$ to another row of $\tilde{A}$, then $\operatorname{det} \tilde{A} \approx \operatorname{det} \tilde{B}$.

Proof. Let $\tilde{B}$ be an interval matrix of order $(n \times n)$ obtained from an interval matrix $\tilde{A}$ of order $(n \times n)$ by adding $k$ times of jth row of $\tilde{A}$ to the ith row of $\tilde{A}$, where $i, j=1,2,3, \ldots, n$. We have

$$
\begin{aligned}
& \tilde{B}=\left(\begin{array}{ccccccc}
\tilde{a}_{11} & \tilde{a}_{12} & \tilde{a}_{13} & \ldots & \ldots & \ldots & \tilde{a}_{1 n} \\
\tilde{a}_{21} & \tilde{a}_{22} & \tilde{a}_{23} & \ldots & \ldots & \ldots & \tilde{a}_{2 n} \\
\ldots & \ldots & \ldots & \ldots & \ldots & \ldots & \ldots \\
\tilde{a}_{j 1} & \tilde{a}_{j 2} & \tilde{a}_{j 3} & \ldots & \ldots & \ldots & \tilde{a}_{j n} \\
\ldots & \ldots & \ldots & \ldots & \ldots & \ldots & \ldots \\
\tilde{a}_{i 1}+k \tilde{a}_{j 1} & \tilde{a}_{i 2}+k \tilde{a}_{j 2} & \tilde{a}_{i 3}+k \tilde{a}_{j 3} & \ldots & \ldots & \ldots & \tilde{a}_{i n}+k \tilde{a}_{j n} \\
\ldots & \ldots & \ldots & \ldots & \ldots & \ldots & \ldots \\
\tilde{a}_{n 1} & \tilde{a}_{n 2} & \tilde{a}_{n 3} & \ldots & \ldots & \ldots & \tilde{a}_{n n}
\end{array}\right) \\
& \text { Now } \operatorname{det} \tilde{B}=\mid \begin{array}{ccccccc}
\tilde{a}_{11} & \tilde{a}_{12} & \tilde{a}_{13} & \ldots & \ldots & \ldots & \tilde{a}_{1 n} \\
\tilde{a}_{21} & \tilde{a}_{22} & \tilde{a}_{23} & \ldots & \ldots & \ldots & \tilde{a}_{2 n} \\
\ldots & \ldots & \ldots & \ldots & \ldots & \ldots & \ldots \\
\tilde{a}_{j 1} & \tilde{a}_{j 2} & \tilde{a}_{j 3} & \ldots & \ldots & \ldots & \tilde{a}_{j n} \\
\ldots & \ldots & \ldots & \ldots & \ldots & \ldots & \ldots \\
\tilde{a}_{i 1}+k \tilde{a}_{j 1} & \tilde{a}_{i 2}+k \tilde{a}_{j 2} & \tilde{a}_{i 3}+k \tilde{a}_{j 3} & \ldots & \ldots & \ldots & \tilde{a}_{i n}+k \tilde{a}_{j n} \\
\ldots & \ldots & \ldots & \ldots & \ldots & \ldots & \ldots \\
\tilde{a}_{n 1} & \tilde{a}_{n 2} & \tilde{a}_{n 3} & \ldots & \ldots & \ldots & \tilde{a}_{n n}
\end{array}
\end{aligned}
$$




$$
\approx\left|\begin{array}{ccccccc}
\tilde{a}_{11} & \tilde{a}_{12} & \tilde{a}_{13} & \ldots & \ldots & \ldots & \tilde{a}_{1 n} \\
\tilde{a}_{21} & \tilde{a}_{22} & \tilde{a}_{23} & \ldots & \ldots & \ldots & \tilde{a}_{2 n} \\
\ldots & \ldots & \ldots & \ldots & \ldots & \ldots & \ldots \\
\tilde{a}_{j 1} & \tilde{a}_{j 2} & \tilde{a}_{j 3} & \ldots & \ldots & \ldots & \tilde{a}_{j n} \\
\ldots & \ldots & \ldots & \ldots & \ldots & \ldots & \ldots \\
\tilde{a}_{i 1} & \tilde{a}_{i 2} & \tilde{a}_{i 3} & \ldots & \ldots & \ldots & \tilde{a}_{i n} \\
\ldots & \ldots & \ldots & \ldots & \ldots & \ldots & \ldots \\
\tilde{a}_{n 1} & \tilde{a}_{n 2} & \tilde{a}_{n 3} & \ldots & \ldots & \ldots & \tilde{a}_{n n}
\end{array}\right|+\left|\begin{array}{ccccccc}
\tilde{a}_{11} & \tilde{a}_{12} & \tilde{a}_{13} & \ldots & \ldots & \ldots & \tilde{a}_{1 n} \\
\tilde{a}_{21} & \tilde{a}_{22} & \tilde{a}_{23} & \ldots & \ldots & \ldots & \tilde{a}_{2 n} \\
\ldots & \ldots & \ldots & \ldots & \ldots & \ldots & \ldots \\
\tilde{a}_{j 1} & \tilde{a}_{j 2} & \tilde{a}_{j 3} & \ldots & \ldots & \ldots & \tilde{a}_{j n} \\
\ldots & \ldots & \ldots & \ldots & \ldots & \ldots & \ldots \\
\tilde{a}_{j 1} & \tilde{a}_{j 2} & \tilde{a}_{j 3} & \ldots & \ldots & \ldots & \tilde{a}_{j n} \\
\ldots & \ldots & \ldots & \ldots & \ldots & \ldots & \ldots \\
\tilde{a}_{n 1} & \tilde{a}_{n 2} & \tilde{a}_{n 3} & \ldots & \ldots & \ldots & \tilde{a}_{n n}
\end{array}\right|
$$

$\approx \operatorname{det} \tilde{A}+k . \tilde{0} \approx \operatorname{det} \tilde{A}$, properties (3) and (5).

Hence $\operatorname{det} \tilde{B} \approx \operatorname{det} \tilde{A}$.

\section{Interval Gaussian Elimination Algorithm}

We propose interval Gaussian elimination algorithm for computing the enclosures of the determinant of interval matrices. For simplicity, we consider a four-by-four square interval matrix

$$
\tilde{A}=\left(\begin{array}{cccc}
\tilde{a}_{11} & \tilde{a}_{12} & \tilde{a}_{13} & \tilde{a}_{14} \\
\tilde{a}_{21} & \tilde{a}_{22} & \tilde{a}_{23} & \tilde{a}_{24} \\
\tilde{a}_{31} & \tilde{a}_{32} & \tilde{a}_{33} & \tilde{a}_{34} \\
\tilde{a}_{41} & \tilde{a}_{42} & \tilde{a}_{43} & \tilde{a}_{44}
\end{array}\right)=\left(\begin{array}{llll}
{\left[\underline{a}_{11}, \bar{a}_{11}\right]} & {\left[\underline{a}_{12}, \bar{a}_{12}\right]} & {\left[\underline{a}_{13}, \bar{a}_{13}\right]} & {\left[\underline{a}_{14}, \bar{a}_{14}\right]} \\
{\left[\underline{a}_{21}, \bar{a}_{21}\right]} & {\left[\underline{a}_{22}, \bar{a}_{22}\right]} & {\left[\underline{a}_{23}, \bar{a}_{23}\right]} & {\left[\underline{a}_{24}, \bar{a}_{24}\right]} \\
{\left[\underline{a}_{31}, \bar{a}_{31}\right]} & {\left[\underline{a}_{32}, \bar{a}_{32}\right]} & {\left[\underline{a}_{33}, \bar{a}_{33}\right]} & {\left[\underline{a}_{34}, \bar{a}_{34}\right]} \\
{\left[\underline{a}_{41}, \bar{a}_{41}\right]} & {\left[\underline{a}_{42}, \bar{a}_{42}\right]} & {\left[\underline{a}_{43}, \bar{a}_{43}\right]} & {\left[\underline{a}_{44}, \bar{a}_{44}\right]}
\end{array}\right)
$$

Steps for reducing the square interval matrix $\tilde{A}$ in to an upper triangular square interval matrix.

Step 1: The Pivot is $\tilde{a}_{11}$

Multiply first row by $\tilde{m}_{21}=-\frac{\tilde{a}_{21}}{\tilde{a}_{11}}$ add the result to second row to get by using dual subtraction and dual division, $\tilde{a}_{21}=\tilde{a}_{21}+\tilde{m}_{21} * \tilde{a}_{11}=\tilde{0} ; \tilde{a}_{22}=$ $\tilde{a}_{22}+\tilde{m}_{21} * \tilde{a}_{12} ; \tilde{a}_{23}=\tilde{a}_{23}+\tilde{m}_{21} * \tilde{a}_{13} ; \tilde{a}_{24}=\tilde{a}_{24}+\tilde{m}_{21} * \tilde{a}_{14}$ Multiply first row by $\tilde{m}_{31}=-\frac{\tilde{a}_{31}}{\tilde{a}_{11}}$ add the result to third row to get $\tilde{a}_{31}=\tilde{0} ; \tilde{a}_{32}=\tilde{a}_{32}+\tilde{m}_{31} *$ $\tilde{a}_{12} ; \tilde{a}_{33}=\tilde{a}_{33}+\tilde{m}_{31} * \tilde{a}_{13} ; \tilde{a}_{34}=\tilde{a}_{34}+\tilde{m}_{31} * \tilde{a}_{14}$ Multiply first row by $\tilde{m}_{41}=-\frac{\tilde{a}_{41}}{\tilde{a}_{11}}$ add the result to fourth row to get $\tilde{a}_{41}=\tilde{0} ; \tilde{a}_{42}=\tilde{a}_{42}+\tilde{m}_{41} * \tilde{a}_{12} ; \tilde{a}_{43}=$ $\tilde{a}_{43}+\tilde{m}_{41} * \tilde{a}_{13} ; \tilde{a}_{44}=\tilde{a}_{44}+\tilde{m}_{41} * \tilde{a}_{14}$

Step 2: The Pivot is $\tilde{a}_{22}$

Multiply second row by $\tilde{m}_{32}=-\frac{\tilde{a}_{32}}{\tilde{a}_{22}}$ add the result to third row to get $\tilde{a}_{31}=$ $\tilde{0} ; \tilde{a}_{32}=\tilde{0} ; \tilde{a}_{33}=\tilde{a}_{33}+\tilde{m}_{32} * \tilde{a}_{23} ; \tilde{a}_{34}=\tilde{a}_{34}+\tilde{m}_{32} * \tilde{a}_{24}$ Multiply second row 
by $\tilde{m}_{42}=-\frac{\tilde{a}_{42}}{\tilde{a}_{22}}$ add the result to fourth row to get $\tilde{a}_{41}=\tilde{0} ; \tilde{a}_{42}=\tilde{0} ; \tilde{a}_{43}=$ $\tilde{a}_{43}+\tilde{m}_{42} * \tilde{a}_{23} ; \tilde{a}_{44}=\tilde{a}_{44}+\tilde{m}_{42} * \tilde{a}_{24}$

Step 3: The Pivot is $\tilde{a}_{33}$

Multiply third row by $\tilde{m}_{43}=-\frac{\tilde{a}_{43}}{\tilde{a}_{33}}$ add the result to fourth row to get $\tilde{a}_{41}=$ $\tilde{0} ; \tilde{a}_{42}=\tilde{0} ; \tilde{a}_{43}=\tilde{0} ; \tilde{a}_{44}=\tilde{a}_{44}+\tilde{m}_{43} * \tilde{a}_{34}$.

The reduced upper triangular square interval matrix is

$$
\tilde{B}=\left|\begin{array}{cccc}
{\left[\underline{a}_{11}, \bar{a}_{11}\right]} & {\left[\underline{a}_{12}, \bar{a}_{12}\right]} & {\left[\underline{a}_{13}, \bar{a}_{13}\right]} & {\left[\underline{a}_{14}, \bar{a}_{14}\right]} \\
{[0,0]} & {\left[\underline{a}_{22}, \bar{a}_{22}\right]} & {\left[\underline{a}_{23}, \bar{a}_{23}\right]} & {\left[\underline{a}_{24}, \bar{a}_{24}\right]} \\
{[0,0]} & {[0,0]} & {\left[\underline{a}_{33}, \bar{a}_{33}\right]} & {\left[\underline{a}_{34}, \bar{a}_{34}\right]} \\
{[0,0]} & {[0,0]} & {[0,0]} & {\left[\underline{a}_{44}, \bar{a}_{44}\right]}
\end{array}\right| .
$$

Now by theorems (4) and (5), we have $|\tilde{A}| \approx|\tilde{B}|$

\section{Interval Gauss Elimination Algorithm Using Int Lab}

A=input('Enter the interval matrix: ')

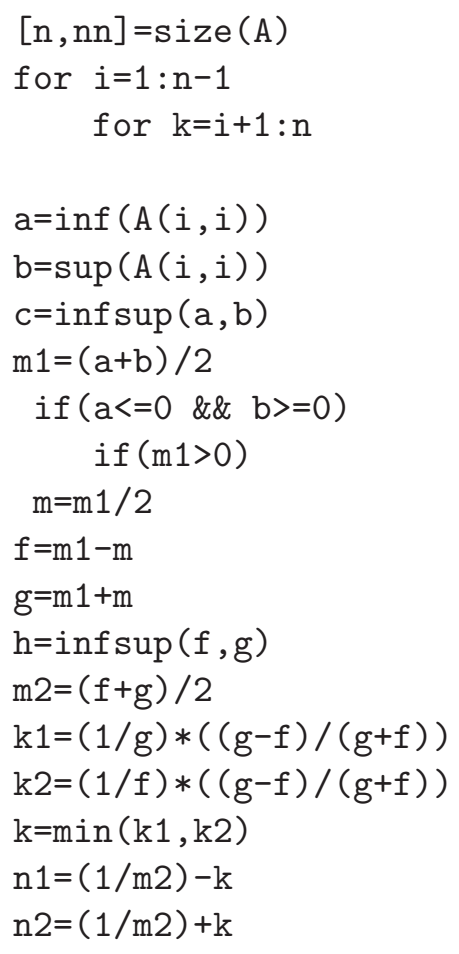




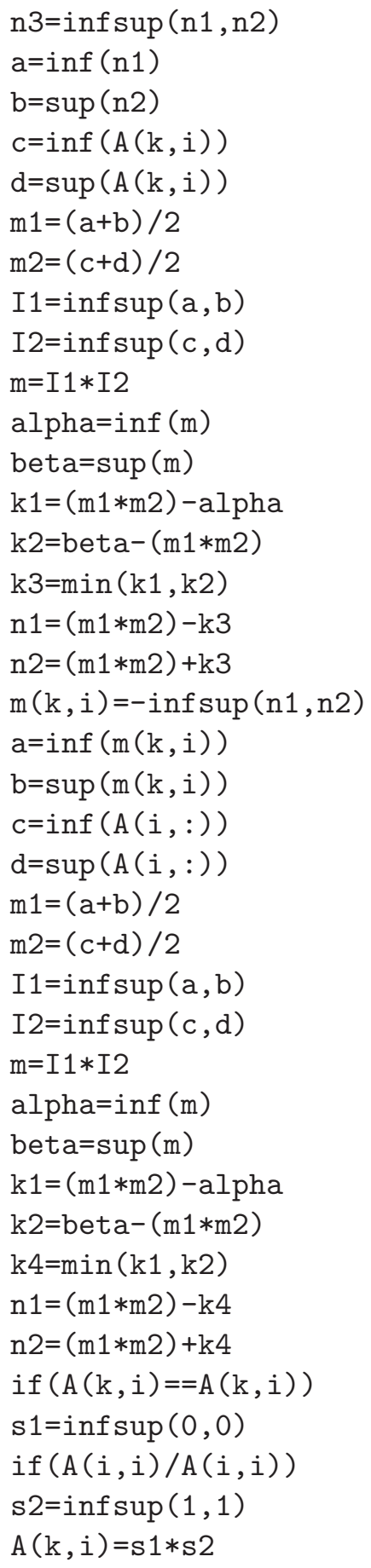




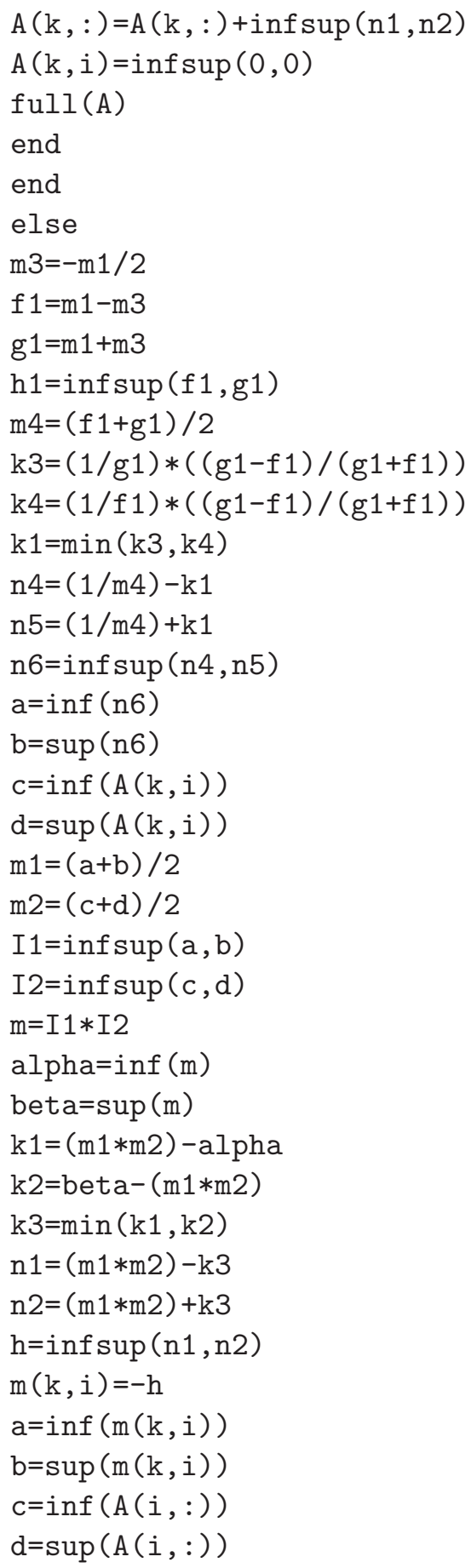




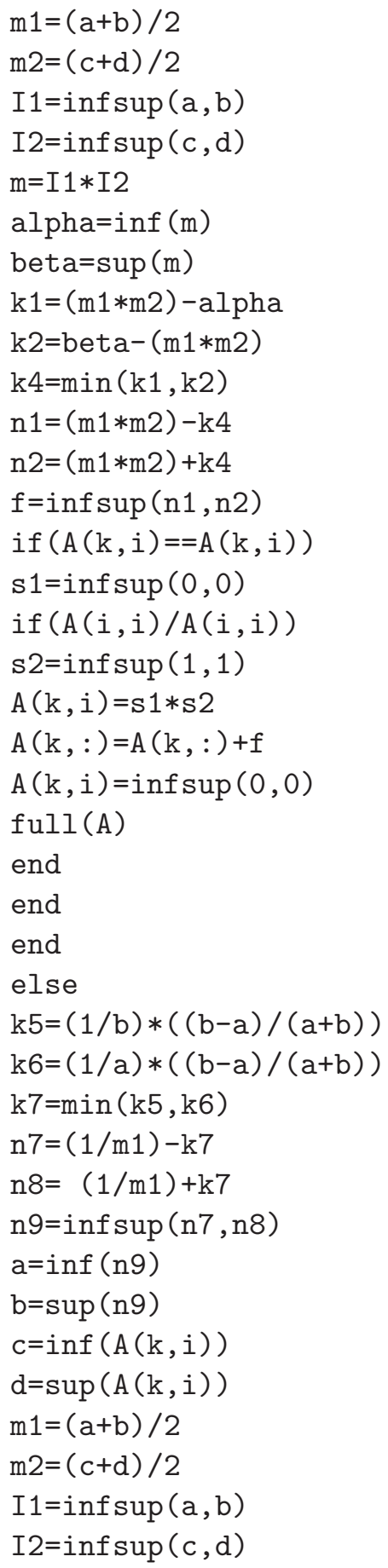




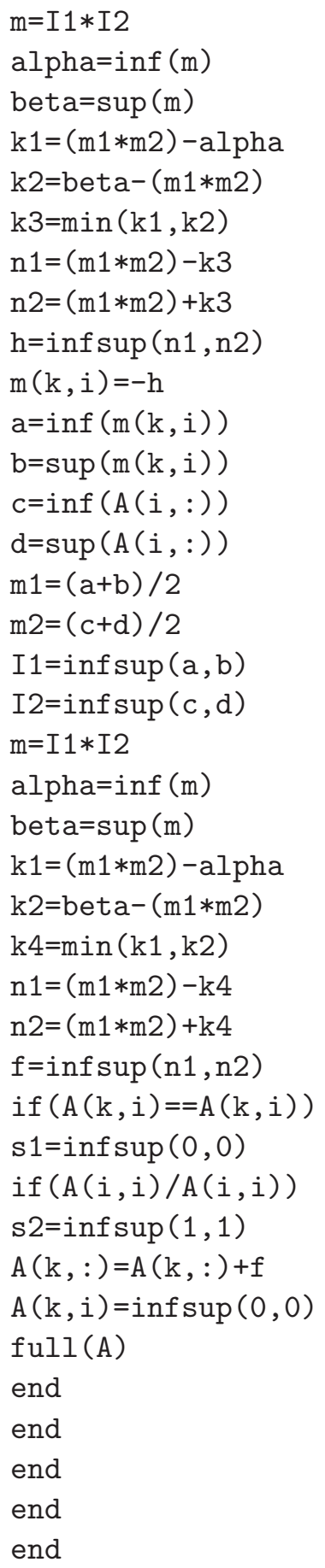




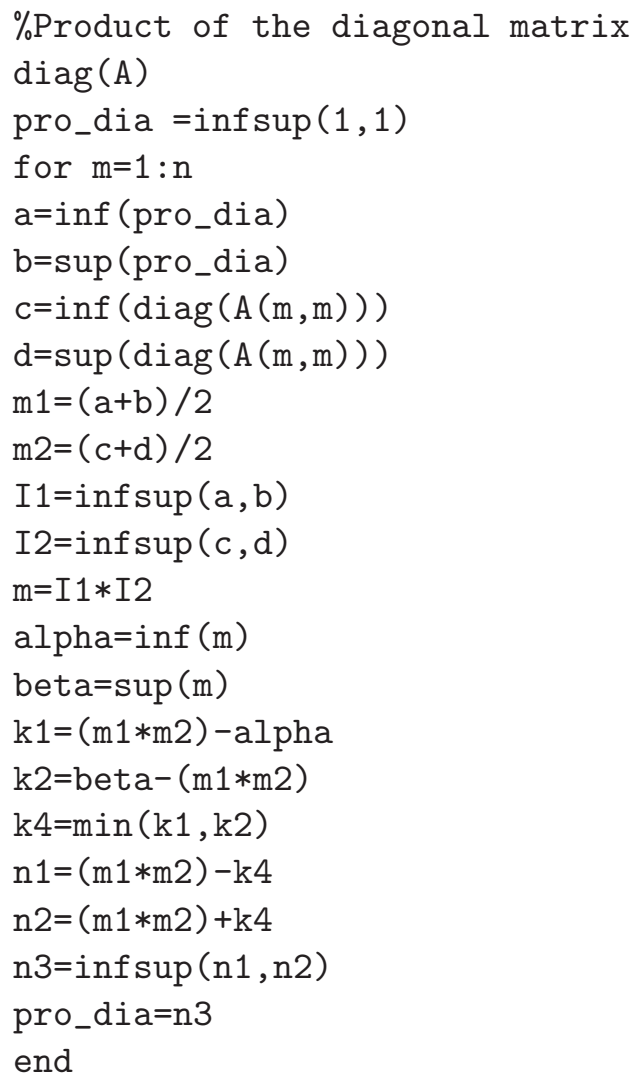

\section{Numerical Examples}

Example 6. Using Gauss elimination algorithm, find the determinant of the interval matrix $\tilde{A}$, where $\tilde{A}=\left(\begin{array}{ccc}{[3.7,4.3]} & {[-1.5,-0.5]} & {[0,0]} \\ {[-1.5,-0.5]} & {[3.7,4.3]} & {[-1.5,-0.5]} \\ {[0,0]} & {[-1.5,-0.5]} & {[3.7,4.3]}\end{array}\right)$,

Step 1: The Pivot element is $\tilde{m}_{21}=[0.1163,0.3837]$

$$
\begin{aligned}
\tilde{a}_{21} & =\tilde{a}_{21}-\frac{\tilde{a}_{21}}{\tilde{a}_{11}} * \tilde{a}_{11} \\
& =[-1.5,-0.5]-\frac{[-1.5,-0.5]}{[3.7,4.3]} *[3.7,4.3] \\
& =\tilde{0} \text { by using dual division and dual subtraction }
\end{aligned}
$$


$\tilde{a}_{22}=[3.2581,4.2419] ; \tilde{a}_{23}=[-1.5,-0.5]$

The reduced matrix is

$$
\left(\begin{array}{ccc}
{[3.7,4.3]} & {[-1.5,-0.5]} & {[0,0]} \\
{[0,0]} & {[3.2581,4.2419]} & {[-1.5,-0.5]} \\
{[0,0]} & {[-1.5,-0.5]} & {[3.7,4.3]}
\end{array}\right)
$$

Step 2: The Pivot element is $\tilde{m}_{32}=[0.1179,0.4154] \tilde{a}_{32}=\tilde{0} ; \tilde{a}_{33}=[3.2256$, 4.2411]. Hence the reduced matrix is

$$
\tilde{B}=\left(\begin{array}{ccc}
{[3.7,4.3]} & {[-1.5,-0.5]} & {[0,0]} \\
{[0,0]} & {[3.2581,4.2419]} & {[-1.5,-0.5]} \\
{[0,0]} & {[0,0]} & {[3.2256,4.2411]}
\end{array}\right) .
$$

Now the determinant of $\tilde{B}$ is the product of the elements in its main diagonal and hence if we evaluate the determinant of the interval matrix $\tilde{A}$ in a straightforward way from the definition, we have

$$
\begin{aligned}
|\tilde{A}| & =\left|\begin{array}{ccc}
{[3.7,4.3]} & {[-1.5,-0.5]} & {[0,0]} \\
{[-1.5,-0.5]} & {[3.7,4.3]} & {[-1.5,-0.5]} \\
{[0,0]} & {[-1.5,-0.5]} & {[3.7,4.3]}
\end{array}\right| \\
& \approx[44.178,75.822]+[-7.075,-0.925] \\
& \approx[37.103,74.897]
\end{aligned}
$$

Here we see that $m(|\tilde{A}|)=m(|\tilde{B}|)=56$. Hence by theorem (4) and (5), we have $|\tilde{A}| \approx[38.8846,73.1159]$.

Example 7. Using Gauss elimination algorithm, find the determinant of the interval matrix $\tilde{A}$, where $\tilde{A}=\left(\begin{array}{cccc}{[4,6]} & {[-1,1]} & {[-1,1]} & {[-1,1]} \\ {[-1,1]} & {[-6,-4]} & {[-1,1]} & {[-1,1]} \\ {[-1,1]} & {[-1,1]} & {[9,11]} & {[-1,1]} \\ {[-1,1]} & {[-1,1]} & {[-1,1]} & {[-11,-9]}\end{array}\right)$

Step 1: The Pivot element is $\tilde{m}_{21}=[-0.2333,0.2333] ; \quad \tilde{a}_{21}=\tilde{0} ; \quad \tilde{a}_{22}=$ $[-6.2334,-3.7666] ; \tilde{a}_{23}=[-1.2334,1.2334] ; \tilde{a}_{24}=[-1.2334,1.2334]$.

The Pivot element is $\tilde{m}_{31}=[-0.2333,0.2333] ; \tilde{a}_{31}=\tilde{0} ; \tilde{a}_{32}=[-1.2334,1.2334]$; $\tilde{a}_{33}=[8.7666,11.2334] ; \tilde{a}_{34}=[-1.2334,1.2334]$.

The Pivot element is $\tilde{m}_{41}=[-0.2334,0.2334] ; \tilde{a}_{41}=\tilde{0} ; \tilde{a}_{42}=[-1.2334,1.2334]$; $\tilde{a}_{43}=[-1.2334,1.2334] ; \tilde{a}_{44}=[-11.2334,-8.7666]$. The reduced matrix is

$$
\left(\begin{array}{cccc}
{[4,6]} & {[-1,1]} & {[-1,1]} & {[-1,1]} \\
{[0,0]} & {[-6.2334,-3.7666]} & {[-1.2334,1.2334]} & {[-1.2334,1.2334]} \\
{[0,0]} & {[-1.2334,1.2334]} & {[8.7666,11.2334]} & {[-1.2334,1.2334]} \\
{[0,0]} & {[-1.2334,1.2334]} & {[-1.2334,1.2334]} & {[-11.2334,-8.7666]}
\end{array}\right) .
$$


Step 2: The Pivot element is $\tilde{m}_{32}=[-0.2955,0.2955] ; \quad \tilde{a}_{31}=\tilde{0} ; \tilde{a}_{32}=\tilde{0}$; $\tilde{a}_{33}=[8.4020,11.5980] ; \tilde{a}_{34}=[-1.5980,1.5980]$.

The Pivot element is $\tilde{m}_{42}=[-0.2955,0.2955] ; \quad \tilde{a}_{41}=\tilde{0} ; \quad \tilde{a}_{42}=\tilde{0} ; \quad \tilde{a}_{43}=$ $[-1.5980,1.5980] ; \tilde{a}_{44}=[-11.5980,-8.4020]$.

The reduced matrix is

$$
\left(\begin{array}{cccc}
{[4,6]} & {[-1,1]} & {[-1,1]} & {[-1,1]} \\
{[0,0]} & {[-6.2334,-3.7666]} & {[-1.2334,1.2334]} & {[-1.2334,1.2334]} \\
{[0,0]} & {[0,0]} & {[8.4020,11.5980]} & {[-1.5980,1.5980]} \\
{[0,0]} & {[0,0]} & {[-1.5980,1.5980]} & {[-11.5980,-8.4020]}
\end{array}\right) .
$$

Step 3: The Pivot element is $\tilde{m}_{43}=[-0.1819,0.1819] ; \quad \tilde{a}_{41}=\tilde{0} ; \quad \tilde{a}_{42}=$ $\tilde{0} ; \tilde{a}_{43}=\tilde{0} ; \tilde{a}_{44}=[-11.8888,-8.1112]$. Hence the reduced upper triangular interval matrix is

$$
\tilde{B}=\left(\begin{array}{cccc}
{[4,6]} & {[-1,1]} & {[-1,1]} & {[-1,1]} \\
{[0,0]} & {[-6.2334,-3.7666]} & {[-1.2334,1.2334]} & {[-1.2334,1.2334]} \\
{[0,0]} & {[0,0]} & {[8.4020,11.5980]} & {[-1.5980,1.5980]} \\
{[0,0]} & {[0,0]} & {[0,0]} & {[-11.8888,-8.1112]}
\end{array}\right)
$$

Now the determinant of $\tilde{B}$ is the product of the elements in its main diagonal and hence $|\tilde{B}|=[4,6] *[-6.2334,-3.7666] *[8.4020,11.5980] *[-11.8888$, $-8.1112] \approx[1026.8,3973.2]$. If we evaluate the determinant of the interval matrix $\tilde{A}$ in a straightforward way from the definition, we have

$$
\begin{aligned}
|\tilde{A}| & =\left|\begin{array}{cccc}
{[4,6]} & {[-1,1]} & {[-1,1]} & {[-1,1]} \\
{[-1,1]} & {[-6,-4]} & {[-1,1]} & {[-1,1]} \\
{[-1,1]} & {[-1,1]} & {[9,11]} & {[-1,1]} \\
{[-1,1]} & {[-1,1]} & {[-1,1]} & {[-11,-9]}
\end{array}\right| \\
& =[868,4132]
\end{aligned}
$$

Here we see that $m(|\tilde{A}|)=m(|\tilde{B}|)=2500$. Hence by theorem (4) and (5), we have $|\tilde{A}| \approx[1026.8,3973.2]$.

\section{Conclusion}

We have defined the concept of determinant for square interval matrices and proved some of its properties. We have proposed an interval Gaussian elimination algorithm for computing the enclosures of determinant of interval matrices. We have solved two numerical examples to show the efficiency of the proposed 
algorithm. It is to be noted that the results obtained by applying the proposed algorithm are sharper (less width)than the results obtained by applying straightforward way from the definition of determinant.

\section{Acknowledgements}

The authors gratefully acknowledge (BRNS - DAE) Board of Research in Nuclear Sciences, Department of Atomic Energy, Government of India for its support through the funded research project grant No.2008/36/35/BRNS/1999 for the investigation presented here.

\section{References}

[1] G. Alefeld and J. Herzberger, Introduction to Interval Computations, Academic Press, New York (1983).

[2] Atanu Sengupta, Tapan Kumar Pal, Theory and Methodology: On comparing interval numbers, European Journal of Operational Research, 27 (2000), 28-43, doi: 10.1016/S0377-2217(99)00319-7.

[3] R. Baker Kearfott, V. Kreinovich, Applications of Interval Computations, Springer (1996).

[4] K. Ganesan, P. Veeramani, On arithmetic operations of interval numbers, International Journal of Uncertainty, Fuzziness and Knowledge - Based Systems, 13, No. 6 (2005), 619-631, doi: 10.1142/S0218488505003710.

[5] K. Ganesan, On some properties of interval matrices, International Journal of Computational and Mathematical Sciences, 1, No. 2 (2007), 92-99, doi: 10.11113.4379.

[6] E.R. Hansen, R.R. Smith, Interval arithmetic in matrix computations, Part 2, SIAM. journal of Numerical Analysis, 4 (1967), 1-9, doi: 10.1137/0704001.

[7] E.R. Hansen, On the solution of linear algebraic equations with interval coefficients, Linear Algebra Appl, 2 (1969), 153-165, doi: 10.1016/00243795 . 
[8] E.R. Hansen, Bounding the solution of interval linear Equations, SIAM Journal of Numerical Analysis, 29, No. 5 (1992), 1493-1503, doi: $10.1137 / 0729086$.

[9] E. Kaucher, Interval analysis in the extended interval space IR, Computing, Suppl., 2 (1980), 33-49, doi: 10.1007/978-3-7091-8577-3.

[10] J.R. Kuttler, A Fourth-order finite-difference approximation for the fixed membrane eigen-problem, Math. Comp., 25 (1971), 237-256, doi: 10.1090/S0025-5718-1971-0301955-6.

[11] Luc Jaulin, Michel Kieffer, Olivier Didrit, Eric Walter, Applied Interval Analysis, Springer-Verlag, London (2001).

[12] R.E. Moore, Methods and Applications of Interval Analysis, SIAM, Philadelphia (1979).

[13] R.E. Moore, R.B. Kearfott, M. Cloud, Introduction to Interval Analysis, SIAM, Philadelphia, January (2009).

[14] A. Neumaier, Interval Methods for Systems of Equations, Cambridge University Press, Cambridge (1990).

[15] S. Ning, R.B. Kearfott, A comparison of some methods for solving linear interval Equations, SIAM Journal of Numerical Analysis, 34 (1997), 12891305, doi: 10.1137/S0036142994270995.

[16] T. Nirmala, D. Datta, H.S. Kushwaha, K. Ganesan, Inverse interval matrix: A new approach, Applied Mathematical Sciences, 5, No. 13 (2011), 607-624, doi: AIML092011005.

[17] J. Rohn, Inverse interval matrix, SIAM Journal of Numerical Analysis, 3 (1993), 864-870, doi: 10.1137/0730044.

[18] Thomas S. Shores, Applied linear Algebra and Matrix Analysis, SpringerVerlag, New York (2007). 